\title{
$r$-process Nucleosynthesis from Three-dimensional Magnetorotational Core-collapse Supernovae
}

\author{
Philipp Mösta ${ }^{1,13}$ (1) , Luke F. Roberts ${ }^{2}$, Goni Halevi ${ }^{1,3}$, Christian D. Ott ${ }^{4,5}$ (D) Jonas Lippuner ${ }^{6,7,8}$ (D), Roland Haas ${ }^{9}$, and \\ Erik Schnetter ${ }^{10,11,12}$ (D) \\ ${ }^{1}$ Department of Astronomy, 501 Campbell Hall \#3411, University of California at Berkeley, Berkeley, CA 94720, USA; pmoesta@berkeley.edu \\ ${ }^{2}$ National Superconducting Cyclotron Laboratory and Department of Physics, Michigan State University, East Lansing, MI, USA \\ ${ }^{3}$ Department of Astrophysical Sciences, Princeton University, Princeton, NJ 08544, USA \\ ${ }^{4}$ TAPIR, California Institute of Technology, Pasadena, CA, USA \\ ${ }^{5}$ Yukawa Institute for Theoretical Physics, Kyoto University, Kyoto, Japan \\ ${ }^{6}$ CCS-2, Los Alamos National Laboratory, P.O. Box 1663, Los Alamos, NM 87545, USA \\ 7 Center for Nonlinear Studies, Los Alamos National Laboratory, P.O. Box 1663, Los Alamos, NM 87545, USA \\ ${ }^{8}$ Center for Theoretical Astrophysics, Los Alamos National Laboratory, P.O. Box 1663, Los Alamos, NM, 87545, USA \\ ${ }^{9}$ NCSA, University of Illinois, Urbana-Champaign, IL, USA \\ ${ }^{10}$ Perimeter Institute for Theoretical Physics, Waterloo, ON, Canada \\ ${ }^{11}$ Department of Physics, University of Guelph, Guelph, ON, Canada \\ ${ }^{12}$ Center for Computation \& Technology, Louisiana State University, Baton Rouge, LA, USA \\ Received 2017 December 30; revised 2018 July 24; accepted 2018 July 24; published 2018 September 13
}

\begin{abstract}
We investigate $r$-process nucleosynthesis in 3D general-relativistic magnetohydrodynamic simulations of rapidly rotating strongly magnetized core collapse. The simulations include a microphysical finite-temperature equation of state and a leakage scheme that captures the overall energetics and lepton number exchange due to postbounce neutrino emission and absorption. We track the composition of the ejected material using the nuclear reaction network SkyNet. Our results show that the 3D dynamics of magnetorotational core-collapse supernovae (CCSN) are important for their nucleosynthetic signature. We find that production of $r$-process material beyond the second peak is reduced by a factor of 100 when the magnetorotational jets produced by the rapidly rotating core undergo a kink instability. Our results indicate that 3D magnetorotationally powered CCSNe are robust $r$-process sources only if they are obtained by the collapse of cores with unrealistically large precollapse magnetic fields of the order of $10^{13} \mathrm{G}$. Additionally, a comparison simulation that we restrict to axisymmetry results in overly optimistic $r$-process production for lower magnetic field strengths.
\end{abstract}

Key words: gamma-ray burst: general - instabilities - magnetohydrodynamics (MHD) - neutrinos - nuclear reactions, nucleosynthesis, abundances - supernovae: general

\section{Introduction}

Magnetorotational core-collapse supernovae (BisnovatyiKogan 1970; LeBlanc \& Wilson 1970; Meier et al. 1976; Wheeler et al. 2002; Mösta et al. 2014a) are promising candidate sites for $r$-process nucleosynthesis (Winteler et al. 2012; Nishimura et al. 2015, 2017b; Halevi \& Mösta 2018). They are also proposed to be the engines driving hyperenergetic supernovae from stripped-envelope progenitors, classified as Type Ic-bl ( $\mathrm{H} / \mathrm{He}$ deficient, broad spectral lines). The amount of ejected $r$-process material has been found to be $\sim 10^{-3}-10^{-2} M_{\odot}$ in previous studies (Winteler et al. 2012; Nishimura et al. 2015), which is similar to what is expected from binary neutron star mergers (e.g., Hotokezaka et al. 2013). Magnetorotational supernovae also have the potential to enrich galaxies early in their cosmic history, as the massive progenitor stars of these explosions live fast and die young. They, therefore, offer an intriguing alternative channel for $r$-process enrichment, especially at low metallicities.

Magnetorotationally driven supernovae require rapid iron core rotation of the progenitor star $\left(P_{0} \simeq \mathcal{O}(1) \mathrm{s}\right)$ (Ott et al. 2006; Burrows et al. 2007; Mösta et al. 2014b) to form an ms-protoneutron star (PNS) after collapse. An additional, magnetar-strength toroidal field then funnels accreted material into a jet propagating along the rotation axis of the star

\footnotetext{
${ }^{13}$ NASA Einstein Fellow.
}

(Meier et al. 1976; Wheeler et al. 2002; Burrows et al. 2007; Mösta et al. 2014b; Obergaulinger \& Aloy 2017). This field can be created by flux-compression from a highly magnetized progenitor core or via amplification by the magnetorotational instability (MRI, Balbus \& Hawley 1991; Akiyama et al. 2003; Obergaulinger et al. 2009) and dynamo action (Mösta et al. 2015 ) in the early postbounce evolution of the PNS.

The thermodynamic conditions in the jet-driven outflows typical for these explosions likely differ from those in neutrinodriven core-collapse supernovae (CCSNe). Material in the outflows is highly magnetized, underdense, and neutron-rich (electron fraction of $Y_{\mathrm{e}} \simeq 0.1-0.3$ ). These are ideal conditions for rapid neutron-capture ( $r$-process) nucleosynthesis (e.g., Hoffman et al. 1997; Meyer \& Brown 1997). Previously, the $r$-process nucleosynthetic signatures of jet-driven CCSNe have been studied with axisymmetric (2D) and 3D magnetohydrodynamic (MHD) simulations. Winteler et al. (2012) found robust $r$-process nucleosynthesis consistent with the solar abundance pattern for a 3D simulation of the collapse of a highly magnetized progenitor core. The simulation exhibited a strong jet explosion that was not disrupted by an $m=1$-kink instability (Mösta et al. 2014b). This was caused by the strong assumed poloidal field $\left(B_{\mathrm{pol}}=5 \times 10^{12} \mathrm{G}\right)$ and stabilized the outflow, since the stability criterion depends on the ratio of toroidal over the poloidal field (Kruskal \& Tuck 1958). The explosion dynamics seen in their simulations are similar to $2 \mathrm{D}$ 
simulations of jet-driven CCSNe (Burrows et al. 2007; Takiwaki et al. 2012). Nishimura et al. (2015) studied the $r$-process nucleosynthetic signatures of a range of 2D axisymmetric MHD CCSN simulations and found that in prompt explosions $\left(t_{\exp } \leqslant 50 \mathrm{~ms}\right)$, a robust $r$-process abundance pattern is recovered, while for delayed explosions, the abundance pattern differs from solar above mass number $A \sim 130$, which includes the second and third $r$-process peaks.

We present results on $r$-process nucleosynthesis from full 3D dynamical-spacetime general-relativistic MHD (GRMHD) simulations of rapidly rotating magnetized CCSNe. We carry out simulations with initial field strengths of $10^{12}$ and $10^{13} \mathrm{G}$ in full unconstrained 3D. For the $10^{12} \mathrm{G}$ case, we compare results with a simulation starting from identical initial conditions but that is set up to remain perfectly axisymmetric in its dynamics. We calculate nucleosynthetic yields by postprocessing Lagrangian tracer particles with the open-source nuclear reaction network SkyNet (Lippuner \& Roberts 2017). We also investigate the impact of neutrinos on the nucleosynthetic yields by varying the uncertain neutrino luminosities from our simulations in the nuclear reaction network calculation.

Our results for a model with an initial poloidal $B$ field of $10^{12} \mathrm{G}$ show that the nucleosynthetic signatures of jet-driven CCSNe are substantially different when simulated in 2D versus $3 \mathrm{D}$. In $2 \mathrm{D}$, robust second and third-peak $r$-process material is synthesized in the explosion, while in full 3D, nuclei beyond the second peak are two orders of magnitude less abundant. Only in a simulation starting with a $10^{13} \mathrm{G}$ poloidal magnetic field (which has dynamics similar to the simulation of Winteler et al. 2012), do we find a robust $r$-process pattern that is consistent with the solar $r$-process residuals. These differences are driven by differing thermodynamic histories of material ejected in the jet. For a $10^{12} \mathrm{G}$ initial magnetic field, we find that ejected material reaches lower maximum density before being ejected than in the simulation with the $10^{13} \mathrm{G}$ field. As a result, this material starts with higher electron fractions as it expands and tries to relax to $\beta$ equilibrium. Additionally, the ejected material in the slower jet explosions experiences more neutrino irradiation, which serves to further increase the electron fraction. Based on this finding, we conclude that only jet-driven CCSNe from already strongly magnetized progenitor star cores $\left(B \simeq 10^{13} \mathrm{G}\right)$ are a viable site for production of the third $r$-process peak. It is, however, unrealistic to expect such strongly magnetized progenitor cores from standard models of massive stellar evolution.

This paper is organized as follows: in Section 2, we present the physical and computational setup and numerical methods used. In Section 3.1, we present the simulation dynamics, followed by a description of the ejecta dynamics in Section 3.2. We discuss the properties of the ejected material in Section 3.3 before concluding with a discussion of our findings in Section 4.

\section{Methods and Setup}

\subsection{Simulations}

We employ ideal GRMHD with adaptive mesh refinement (AMR) and spacetime evolution provided by the open-source Einstein Toolkit (Löffler et al. 2012; Mösta et al. 2014a). GRMHD is implemented in a finite-volume fashion with WENO5 reconstruction (Tchekhovskoy et al. 2007; Reisswig et al. 2013) and the HLLE Riemann solver (Einfeldt 1988) and constrained transport (Tóth 2000) for maintaining $\operatorname{div} B=0$. We employ the $K_{0}=220 \mathrm{MeV}$ variant of the equation of state of Lattimer \& Swesty (1991) and the neutrino leakage/heating approximations described in O'Connor \& Ott (2010) and Ott et al. (2012). At the precollapse stage, we cover the inner $\sim 5700 \mathrm{~km}$ of the star with four AMR levels in a Cartesian grid and add five more during collapse. After bounce, the PNS is covered with a resolution of $\sim 370 \mathrm{~m}$, and the AMR grid structures consists of boxes with extents $5674.0 \mathrm{~km}, 3026.1 \mathrm{~km}$, $2435.1 \mathrm{~km}, 1560.3 \mathrm{~km}, 283.7 \mathrm{~km}, 212.8 \mathrm{~km}, 144.8 \mathrm{~km}, 59.1 \mathrm{~km}$, and $17.7 \mathrm{~km}$. The coarsest resolution is $h=94.6 \mathrm{~km}$, and refined meshes differ in resolution by factors of two. We use adaptive shock tracking to ensure that the shocked region is always contained on the mesh refinement box with a resolution of $h=1.48 \mathrm{~km}$.

We draw the $25 M_{\odot}$ (at zero-age-main-sequence) presupernova model E25 from Heger et al. (2000). While this model includes rotation, we parameterize the initial rotation law to match the simulations in Mösta et al. (2014b). The rotation law is cylindrical and axisymmetric following Takiwaki \& Kotake (2011, their Equation (1)) and as in Mösta et al. (2014b):

$$
\Omega(x, z)=\Omega_{0} \frac{x_{0}^{2}}{x^{2}+x_{0}^{2}} \frac{z_{0}^{4}}{z^{4}+z_{0}^{4}},
$$

with an initial central angular velocity of $\Omega_{0}=2.8 \mathrm{rad} \mathrm{s}^{-1}$. The fall-off of the angular velocity profile with the cylindrical radius and vertical position is controlled by parameters $x_{0}=$ $500 \mathrm{~km}$ and $z_{0}=2000 \mathrm{~km}$, respectively. The rotation parameter of our setup is $\beta_{\text {rot }}=0.1 \%$ where $\beta_{0} \equiv T /|W|$ is the ratio of kinetic and gravitational potential energy. This is consistent with typical GRB-oriented progenitors models (i.e., E25 in Heger et al. 2000 has $\beta_{\text {rot }} \sim 0.15 \%$ ). We set up the initial magnetic field using a vector potential of the form

$$
A_{r}=A_{\theta}=0 ; A_{\phi}=B_{0}\left(r_{0}^{3}\right)\left(r^{3}+r_{0}^{3}\right)^{-1} r \sin \theta,
$$

where $B_{0}$ controls the strength of the field. In this way, we obtain a modified dipolar field structure that stays nearly uniform in strength within radius $r_{0}$ and falls off like a dipole at larger radii. We choose $r_{0}=1000 \mathrm{~km}$ to match the initial conditions of model B12X5 $\beta 0.1$ of the 2D study of Takiwaki $\&$ Kotake (2011) and the 3D study of Mösta et al. (2014b). We perform simulations for two different initial magnetic field strengths, $B_{0}=10^{13} \mathrm{G}$ (B13 from here on) and $B_{0}=10^{12} \mathrm{G}$ (B12). While specific constraints on core magnetic fields of massive stars are not available from observations or multi-D stellar evolution calculations, we deem model B13 with its ultra-strong poloidal precollapse field to be likely unrealistic. B12 and B12-sym have nearly equally high precollapse fields, but Mösta et al. (2015) have shown that the resulting $10^{15} \mathrm{G}$ field after core bounce can be delivered by the MRI and dynamo action. We, therefore, consider these models to be more realistic.

We perform simulations in full, unconstrained 3D. For model B12, we add random perturbations with a magnitude of $1 \%$ of the velocity at the start of the simulation. In model B12sym, we do not add perturbations, and the simulation, therefore, evolves identical to an octant symmetry $3 \mathrm{D}\left(90^{\circ}\right.$ rotational symmetry in the $x-y$ plane and reflection symmetry across the $x-y$ plane) simulation. In this way, we reproduce the dynamics of an axisymmetric simulation while keeping the 
Table 1

Initial Magnetic Field Strength and Perturbation Setup (in Velocity) for the Three Simulations Considered here

\begin{tabular}{lccc}
\hline \hline Simulation & B13 & B12-sym & B12 \\
\hline$B_{\text {pol }}(\mathrm{G})$ & $10^{13}$ & $10^{12}$ & $10^{12}$ \\
Perturbations & None & None & $0.01 \times|\boldsymbol{v}|$ \\
\hline
\end{tabular}

tracer set up and distribution identical between the simulations. In model B13, the 10 times stronger initial poloidal magnetic field prevents the disruption of the jet by a kink instability (Mösta et al. 2014b), as the key quantity for instability is the ratio of toroidal over poloidal field (see Equation (2) in Mösta et al. 2014b). As a result, simulations with and without perturbations for model B13 are nearly identical, and we only present results for a simulation without added perturbations. Model B13 is closest in dynamics to the model presented in Winteler et al. (2012), while model B12-sym mimics the dynamics of the prompt (an explosion within $50 \mathrm{~ms}$ after core bounce) 2D jet explosions in Takiwaki \& Kotake (2011) and Nishimura et al. (2015). We summarize the initial magnetic field strengths and perturbation setups used in the simulations in Table 1.

\subsection{Tracer Particles and Postprocessing}

We extract the thermodynamic conditions of ejected material using Lagrangian tracer particles. We place $10^{5}$ tracer particles on each of the simulations. We limit particles to $30 \mathrm{~km} \leqslant$ $r \leqslant 1000 \mathrm{~km}$ for all simulations to ensure high enough resolution in the particle mass but also guarantee that the infall time for the outermost shell of particles is longer than the simulated time. The tracers are uniformly spaced, so that they represent regions of a constant volume. Each tracer particle gets assigned a mass, taking into account the density at its location and the volume the particle covers. Tracer particles are advected passively with the fluid flow, and data from the 3D simulation grid are interpolated to the tracer particle positions. In this way, we record the thermodynamic conditions and neutrino luminosities the particles encounter as a function of time. To determine the ejected mass in the explosion, we only take dynamically unbound particles into account. We determine whether a particle is unbound by determining the total specific energy is positive (and define the specific energy as the sum of internal, kinetic, and magnetic energy).

For models B13 and B12-sym, we map the tracer particle distribution onto the simulation shortly after core bounce, as both of these models explode within the first $40 \mathrm{~ms}$ of postbounce evolution. Model B12 takes considerably longer to explode, and we map the tracer distribution onto the simulation shortly before the transition to an explosion at time $t-t_{\text {bounce }}=80 \mathrm{~ms}$. This allows us to ensure that we have a sufficient number of tracer particles in the outflows along the rotation axis of the core. We postprocess the particles with the open-source nuclear reaction network SkyNet of Lippuner \& Roberts (2017). The network includes 7843 isotopes up to isotope ${ }^{337} \mathrm{Cn}$. Forward strong rates are taken from the JINA REACLIB database (Cyburt et al. 2010), and inverse rates are computed assuming detailed balance. Weak rates are taken from Fuller et al. (1982), Oda et al. (1994), Langanke \& Martínez-Pinedo (2000), or otherwise from REACLIB. REACLIB also provides nuclear masses and partition functions. SkyNet evolves the temperature via the computation of source terms due to the individual nuclear reactions and neutrino interactions.

Computations for each particle start from nuclear statistical equilibrium (NSE). We start the network as soon as the temperature drops below $T=25 \mathrm{GK}$. The initial conditions for the network calculation are taken at this time. The neutrino luminosity data from the particle trajectories are noisy due to interpolation effects and the very high time resolution at which the tracer particles record the neutrino luminosities. We, therefore, compute a movingwindow time average of the neutrino luminosities of the form $\nu_{\mathrm{av}, i}=\alpha \cdot \bar{\nu}_{i}+(1.0-\alpha) \cdot \nu_{\mathrm{av}, i-1}$, where $i$ denotes the current timestep data and $i-1$ is the previous one. We choose a weight function for each data set in the moving average as $\alpha=$ $2 \cdot(n+1.0)^{-1}$, with $n=40$, and keep the neutrino luminosities constant after the end of the particle data. In cases in which the particle data in the simulation does not reach temperatures low enough for the network calculation to start, we extrapolate the particle data assuming homologous expansion. We carry out the network calculations to $10^{9} \mathrm{~s}$, which is sufficient to generate stable abundance patterns as a function of mass number $A$.

\section{Results}

\subsection{MHD Dynamics}

Collapse and early postbounce evolution proceed identically in B12 and B12-sym. Core bounce occurs $\sim 350 \mathrm{~ms}$ after the onset of collapse for model B12 and $\sim 450 \mathrm{~ms}$ for model B13. The delay in core bounce for model B13 is due to the additional support by the 100 times higher magnetic pressure. Shortly after core bounce, the poloidal and toroidal B-field components reach $B_{\text {pol }}, B_{\text {tor }} \sim 10^{15} \mathrm{G}$ for model $\mathrm{B} 12$ and $B_{\mathrm{pol}}, B_{\mathrm{tor}} \sim$ $10^{16} \mathrm{G}$ for $\mathrm{B} 13$.

In model B13, the hydrodynamic shock launched at bounce, while still approximately spherical, never stalls, but continues to propagate into a jet explosion along the rotation axis (see left panels of Figures 1 and 2). The jet is powered by the extra pressure and stress from the strong magnetic field. The shock propagates at mildly relativistic speeds $\left(v_{\text {jet }} \sim 0.1-0.2 c\right)$ and reaches $1000 \mathrm{~km}$ at around $35 \mathrm{~ms}$ after core bounce. The jet is stabilized against the MHD kink instability by its large poloidal magnetic field (see stability condition Equation (2) in Mösta et al. 2014b). A mild $m=0$ deformation is visible in the outflow in Figure 2.

In models B12 and B12-sym, the bounce shock stalls after $\sim 10 \mathrm{~ms}$ at a radius of $\sim 110 \mathrm{~km}$. At this time, there is strong differential rotation in the region between the PNS core and the shock. This differential rotation powers rotational winding of the magnetic field and amplifies its toroidal component to $10^{16} \mathrm{G}$ near the rotation axis within $20 \mathrm{~ms}$ of bounce. The strong polar magnetic pressure gradient, in combination with hoop stresses exerted by the toroidal field, then launches a bipolar outflow. As in Mösta et al. (2014b), B12-sym now continues into a jet explosion and reaches $\sim 900 \mathrm{~km}$ after $\sim 100 \mathrm{~ms}$. The expansion speed at that point is mildly relativistic $\left(v_{\text {jet }} \simeq 0.1-0.15 c\right)$.

The 3D simulation with perturbations B12 starts to diverge from its symmetric counterpart B12-sym around $\sim 15 \mathrm{~ms}$ after bounce due to the non-axisymmetric spiral MHD kink instability (Mösta et al. 2014b). The subsequent 3D evolution 

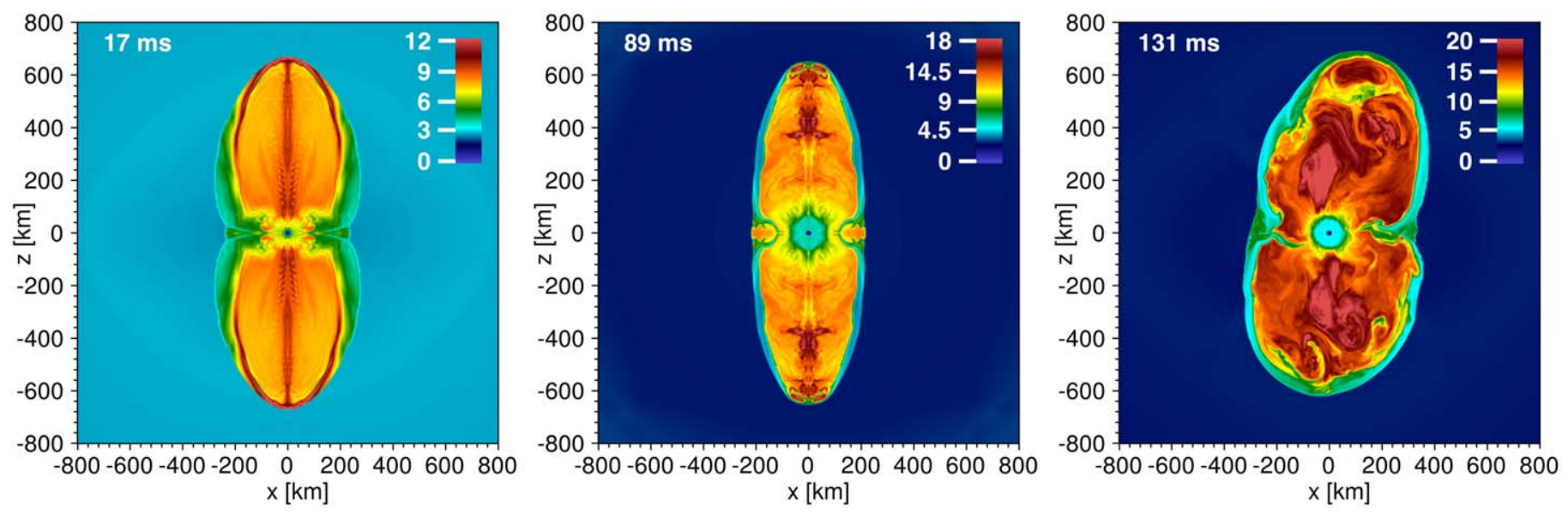

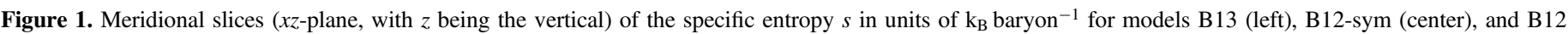

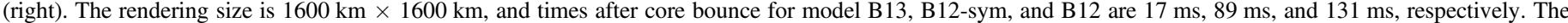

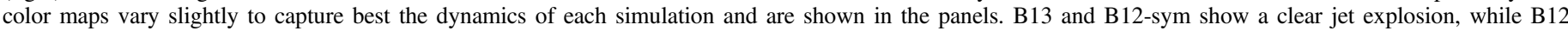
explodes in a dual-lobe fashion due to the jet's kink instability (Mösta et al. 2014b).

is fundamentally different from both the effectively axisymmetric B12-sym model and model B13. The jet is strongly disrupted by the kink instability, which causes the outflow to cover a larger solid angle. The shock also propagates at a lower velocity than in simulations B13 and B12-sym $\left(v_{\text {jet }} \simeq\right.$ $0.03-0.05 c$ ).

The developed jet structures of models B13, B12-sym, and B12 are depicted in Figure 1 (meridional slices) and Figure 2 (volume renderings). In both B13 (left) and B12-sym (center), a clean jet emerges and propagates at mildly relativistic speeds into the outer layers of the core and star. For simulation B12, the explosion propagates in a dual-lobe fashion, as in Mösta et al. (2014b), at nonrelativistic speeds. Material in the outflows of all three simulations is highly magnetized $\left(\beta=P_{\mathrm{gas}} / P_{\mathrm{mag}}\right.$ $\ll 1$ ), ranges between $10 k_{\mathrm{B}}$ baryon $^{-1} \leqslant s \leqslant 20 k_{\mathrm{B}}$ baryon $^{-1}$ in specific entropy, and is neutron-rich $\left(0.1 \leqslant Y_{\mathrm{e}} \leqslant 0.4\right)$.

\subsection{Ejecta Dynamics}

Material that gets accreted across the shock into the postshock flow is pushed to higher densities and temperatures as it is advected toward the PNS. Eventually, some of this infalling material is entrained in the outflow and ejected. We show the evolution of the temperature and density for a typical tracer particle trajectory from simulation B13 in Figure 3. Additionally, we show neutrino luminosities for both electron and electron antineutrinos in Figure 4, as recorded by three representative particles from simulations B13, B12-sym, and B12. After the initial neutronization burst that is visible for simulations B13 and B12-sym in the first $20 \mathrm{~ms}$ of postbounce evolution, the neutrino luminosities for both electron neutrinos and antielectron neutrinos converge toward values of $\simeq 5 \times$ $10^{52} \mathrm{erg} \mathrm{s}^{-1}$ and stay approximately constant for the duration of the simulations.

Material that is ejected often reaches conditions where weak reactions proceed rapidly enough for weak equilibrium (or $\beta$ equilibrium) to nearly take hold. In weak or $\beta$ equilibrium, the rate of neutron destruction balances the rate of proton destruction. For a closed, thermalized system, $\beta$ equilibrium is characterized by the condition $\mu_{\nu_{e}}+\mu_{n}=\mu_{e}+\mu_{p}$, where $\mu_{i}$ is the chemical potentials of electron neutrinos, neutrons, electrons, and protons, respectively. For a fixed lepton fraction $Y_{L}=Y_{\mathrm{e}}+Y_{\nu_{\mathrm{e}}}$, the condition of $\beta$ equilibrium determines $Y_{\mathrm{e}}$ and the net electron neutrino fraction, $Y_{\nu_{\mathrm{e}}}$. When neutrinos are not trapped, the material moves toward dynamic $\beta$ equilibrium, which is not determined by chemical potential equality but rather by rate balance (e.g., Arcones et al. 2010). Considering only captures on neutrons and protons, dynamic $\beta$ equilibrium is given by the condition

$$
\begin{aligned}
\dot{Y}_{\mathrm{e}}= & {\left[\lambda_{e}+\left(\rho, T, Y_{e}\right)+\lambda_{\bar{\nu}_{e}}\right] Y_{n} } \\
& -\left[\lambda_{e}-\left(\rho, T, Y_{e}\right)+\lambda_{\nu_{e}}\right] Y_{p}=0,
\end{aligned}
$$

where the free proton and neutron fractions are set by NSE, and $\lambda_{\mathrm{e}-}, \lambda_{\mathrm{e}+}, \lambda_{\nu_{\mathrm{e}}}$, and $\lambda_{\bar{\nu}_{\mathrm{e}}}$ are the rates of electron, positron, electron neutrino, and electron antineutrino capture, respectively. Equation (2) is an implicit equation for $Y_{\mathrm{e}}$ in $\beta$ equilibrium, $Y_{\mathrm{e}, \beta}=Y_{\mathrm{e}, \beta}(\rho, T)$. When material is out of $\beta$ equilibrium, weak interactions will push its $Y_{\mathrm{e}}$ toward $Y_{\mathrm{e}, \beta}$ on a timescale given by $\tau_{\text {weak }}=\left(\lambda_{e}-\left(\rho, T, Y_{e}\right)+\lambda_{e}+\left(\rho, T, Y_{e}\right)+\lambda_{\nu_{e}}+\lambda_{\bar{\nu}_{e}}\right)^{-1}$. If $\tau_{\text {weak }}$ is shorter than the dynamical timescale, $\tau_{d}=\rho / \dot{\rho}$, then the material should relax to a composition determined by $Y_{e, \beta}$.

The value of $Y_{\mathrm{e}, \beta}$ depends both on the imposed neutrino fluxes and the thermodynamic state of the material, which determines the lepton capture rates. Generally, the electron capture rate dominates at high densities where electrons are degenerate, which pushes $Y_{\mathrm{e}, \beta}$ to values less than $\sim 0.3$. For fixed entropy at densities between $10^{9}$ and $10^{12} \mathrm{~g} \mathrm{~cm}^{-3}$, the $\beta$-equilibrium electron fraction goes down with the increasing density. Therefore, rapidly ejected material that has reached a higher density will often have a lower $Y_{\mathrm{e}}$ at the time that $r$-process nucleosynthesis begins because it experienced more electron captures. On the other hand, when neutrino captures dominate (and when there are no nuclei present), one finds $Y_{\mathrm{e}, \beta} \approx \lambda_{\nu_{\mathrm{e}}}\left(\lambda_{\bar{\nu}_{\mathrm{e}}}+\lambda_{\nu_{\mathrm{e}}}\right)^{-1}$ (Qian \& Woosley 1996). Since the neutrino emission in CCSNe is fairly similar in all flavors, $\beta$ equilibrium driven by neutrino captures generally predicts $Y_{\mathrm{e}, \beta}>0.4$. Material ejected in the jet goes through density regimes where the electron captures dominate and then later through regimes where the neutrino captures dominate. In many cases, the material is never able to fully attain $\beta$ equilibrium.

In the jet-driven $\mathrm{SNe}$ considered here, weak reactions at high density play a dominant role in setting $Y_{\mathrm{e}}$ just before nucleosynthesis starts. This in turn greatly impacts nucleosynthesis in the 

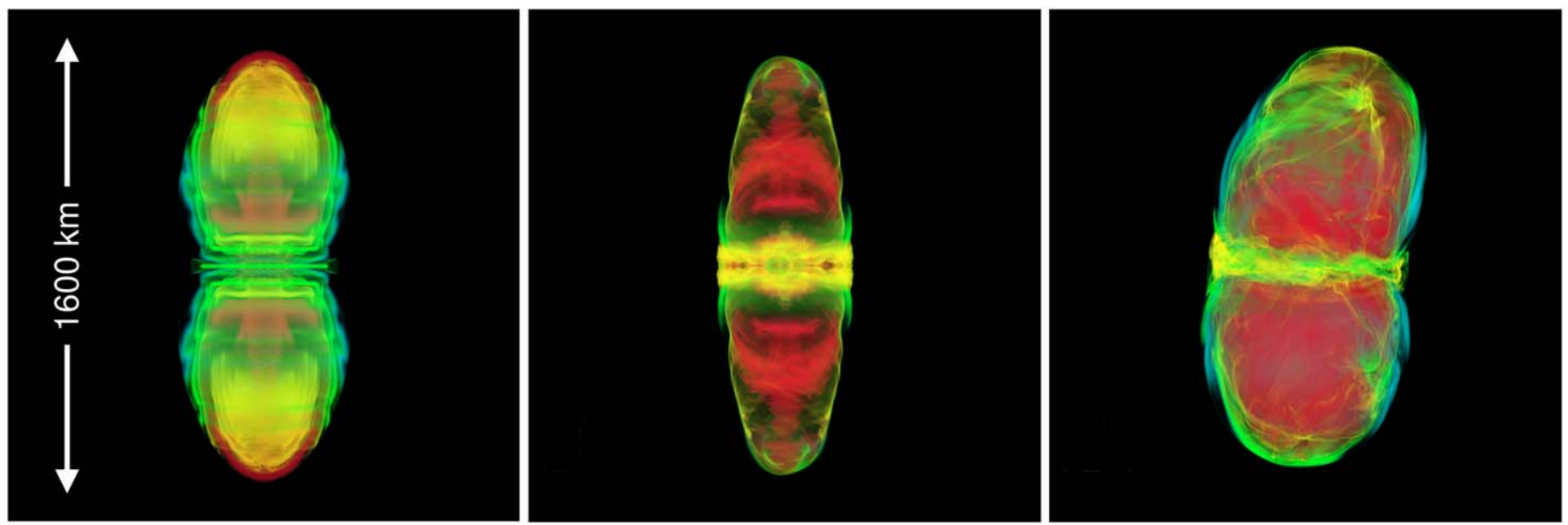

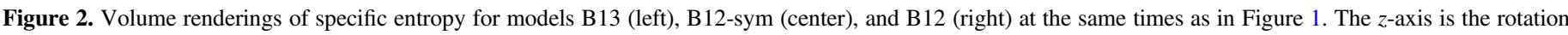

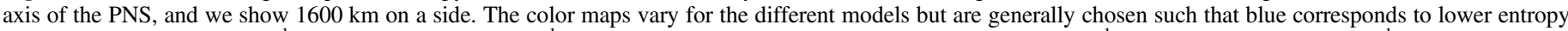

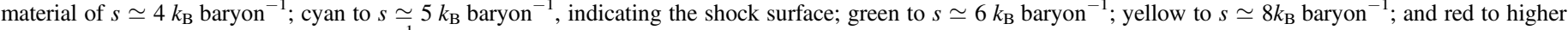
entropy material at $s \simeq 10-12 k_{\mathrm{B}}$ baryon $^{-1}$.

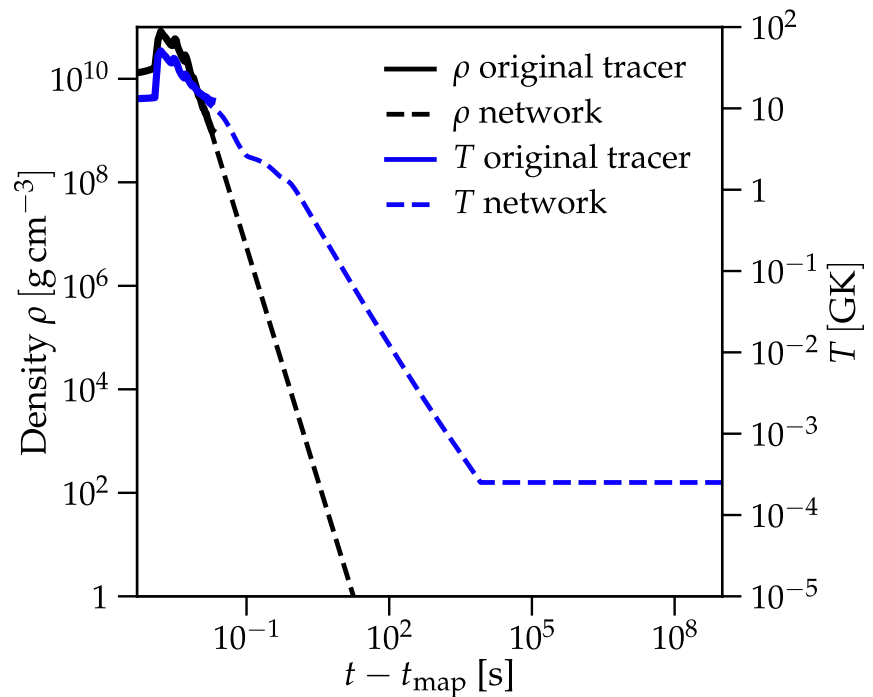

Figure 3. Density $\rho$ (black) and temperature (blue) for a representative tracer for simulation B13. Solid lines at early times show the original tracer particle data, while dashed lines at later times show data extrapolated by the network under the assumption of a homologous expansion. The network expands the particle with $\rho(t)=\rho_{0} \cdot t^{-3}$ and $T(t)=T_{0} \cdot t^{-1}$ until a minimum temperature is reached.

ejecta, since the electron fraction is the determining factor in whether or not a robust $r$-process occurs. In particular, the dynamics of the MHD explosion influence the conditions under which the electron fraction of ejected material is set, since the dynamics determine when $\tau_{\text {weak }}$ becomes close to the dynamical timescale and when weak interactions freeze out. In Figure 5, we show the evolution of $Y_{\mathrm{e}}, Y_{\mathrm{e}, \beta}, \tau_{\text {weak }}$, and $\tau_{d}$ for representative particles from the three simulations. The four colored lines indicate the $Y_{\mathrm{e}}$ evolution as obtained from the nuclear reaction network calculation using four constant neutrino luminosities. In these calculations, we assume $L_{\nu_{e}}=L_{\nu_{\bar{e}}}$ and constant mean neutrino energies of $\left\langle\epsilon_{\nu_{e}}\right\rangle=10 \mathrm{MeV}$ and $\left\langle\epsilon_{\nu_{e}}\right\rangle=14 \mathrm{MeV}$. The solid black lines show the $Y_{e}$ evolution using the neutrino luminosities, as obtained from the tracer particles. There is an initial decrease in $Y_{\mathrm{e}}$ as material is advected inward to higher density, causing $Y_{e, e q}$ to go down and $\tau_{\text {weak }}$ to decrease. Except in a limited number of cases, $\tau_{d}<\tau_{\text {weak }}$ and $\beta$ equilibrium is never obtained, although $Y_{e}$ is always moving toward $Y_{e, \beta}$. Then, as the particle moves outward with the jet and evolves toward lower densities, the electron capture rate is reduced, and neutrino captures begin to dominate the weak reaction rates, causing $Y_{e}$ to increase (this occurs before $0.1 \mathrm{~s}$ in all of the plots). Finally, once the temperature reaches $T \approx 5 \mathrm{GK}$, $r$-process nucleosynthesis begins and $\beta^{-}$-decays of heavy nuclei cause $Y_{e}$ to increase. The initial value of $Y_{e}$ that is relevant at the start of $r$-process nucleosynthesis is seen as the plateau near $1 \mathrm{~s}$ in Figure 5 .

In the most energetic model, B13, the jet is formed at high densities very soon after bounce. As the jet propagates out, it entrained collapsing material that has not fully deleptonized, trapped neutrinos, and reached weak equilibrium. Therefore, after the ejecta begins to move out to larger radii and smaller densities, its $Y_{e}$ still goes down due to electron captures trying to move the material toward the lower $Y_{e}$ predicted by neutrinofree $\beta$ equilibrium. The particles in B13 do not reach their minimum $Y_{e}$ at their maximum density, but rather continue to experience electron captures that drive $Y_{e}$ down toward its neutrino-free $\beta$-equilibrium value. This generally pushes the ejected material in B13 to small $Y_{e}$ values $\left(Y_{e} \simeq 0.15\right)$.

In model B12-sym, the evolution differs since it takes $\simeq 20-30 \mathrm{~ms}$ before a jet explosion is launched, and the propagation of the jet is slower than in model B13. Tracer particles that accrete toward the PNS reach slightly lower maximum densities than in simulation B13, but these particles reach their lowest $Y_{e}$ at their maximum density. As particles get ejected in the outflow, they evolve to higher $Y_{e}$ due to neutrino interactions. This effect is more pronounced for higher neutrino luminosities and is enhanced compared to simulation B13 due to the longer dwell time of particles in the vicinity of the PNS.

In model B12, the explosion dynamics are drastically different than in models B13 and B12-sym. The shock starts to expand only after $\simeq 80 \mathrm{~ms}$. Additionally, particles do not accrete to minimum radii as low as in simulations B13 and B12-sym, hence they reach smaller maximum densities before being ejected. The eventually ejected material comes close to reaching $\beta$ equilibrium when it reaches its maximum density. Since this maximum density is lower than the maximum densities encountered in B12-sym, the minimum $Y_{e}$ reached by 

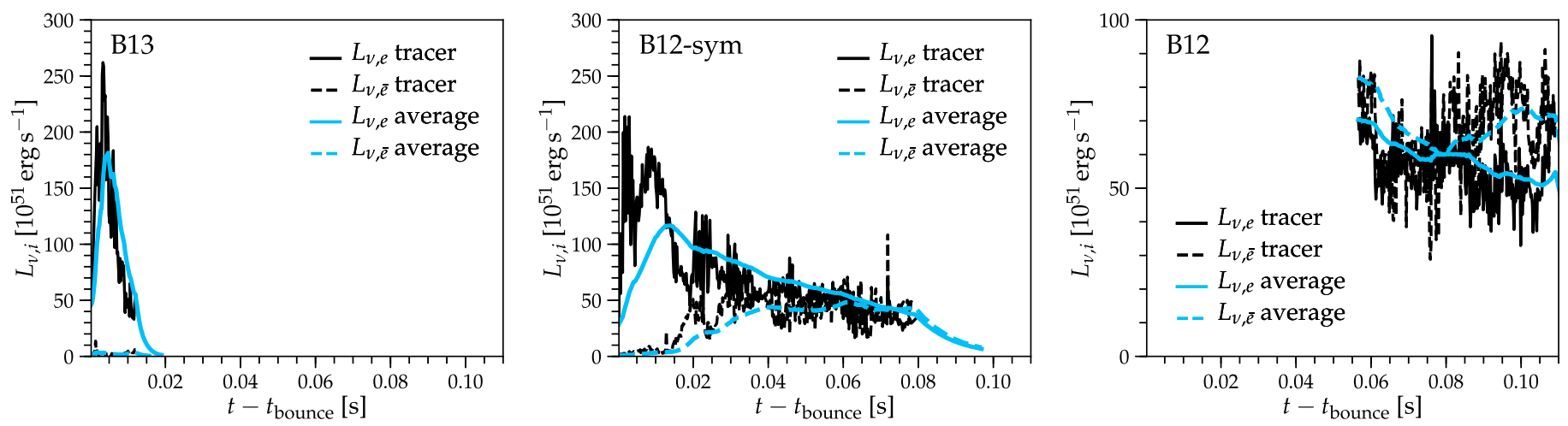

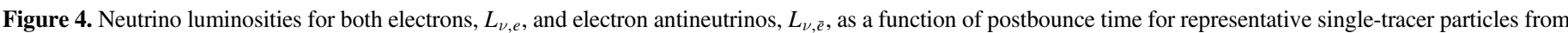

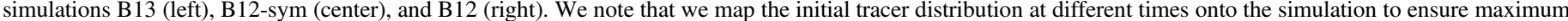

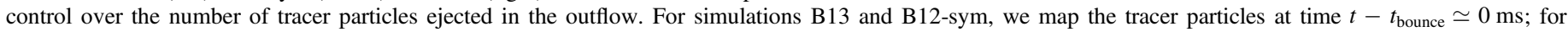
simulation $\mathrm{B} 12$, we map at $t-t_{\text {bounce }} \simeq 80 \mathrm{~ms}$.

the ejected B12 material is systematically higher. The propagation speed of the explosion is slower than in models B13 and B12-sym. However, the dwell time of particles in the vicinity of the PNS before being ejected is similar to that of model B12-sym. This is due to a similar ejection speed in the initially forming outflow near the PNS. It is only the shock surface itself that propagates at slower expansion speeds as the outflow material spirals away from the rotation axis. As the ejected material interacts with neutrinos, it evolves to higher $Y_{e}$ values. This rise in $Y_{e}$ is similar to the evolution in simulation B12-sym.

We show selected particles from simulations B13, B12-sym, and $\mathrm{B} 12$ as a scatter plot in $Y_{e}$ at $T=5 \mathrm{GK}$ and specific entropy in Figure 6. This figure illustrates the behavior described above for the individual tracer particles mentioned above. The symbols for each particle are color coded with the maximum density reached. For simulation B13, particles reach the highest densities as they reach the smallest minimum radii. The $Y_{e}$ values at the time when the particles last exceed a temperature of $5 \mathrm{GK}$ (approximately the temperature threshold for $r$-process nucleosynthesis) are peaked at low $Y_{e} \simeq 0.2$. The entropy values for the particles are similar to those for simulation B12-sym but are lower than for simulation B12. The low $Y_{e}$ values for simulation B13 are almost exclusively set by $\beta$ equilibrium since neutrino irradiation has less of an effect on the $Y_{e}$ distribution, even for high neutrino luminosities, since material gets ejected very rapidly and efficiently. In model B12-sym, particles are at similar entropy but at lower maximum densities and higher $Y_{e}$ values at $T=5 \mathrm{GK}$ compared to simulation B13. The $Y_{e}$ values at $T=5 \mathrm{GK}$ for the particles from simulation B12-sym are set by two effects. First, the $\beta$-equilibrium $Y_{e}$ values for these particles at lower densities are higher than for the particles at higher densities in simulation B13. Second, the dwell time for particles in the vicinity of the PNS is an order of magnitude longer in simulation B12-sym than in B13. This causes the $Y_{e}$ values of the particles to shift to higher values as they cool to $T=5 \mathrm{GK}$. In the full 3D simulation B12, particles are at the lowest maximum densities and highest entropies. Their $Y_{e}$ values in $\beta$ equilibrium are, therefore, higher than for both B13 and B12-sym. The shift in the $Y_{e}$ distribution as the particles evolve toward $T \simeq 5 \mathrm{GK}$ is similar to the evolution in simulation B12-sym. This is caused by the similar dwell time of material at small radii before being ejected in the outflow and hence a similar amount of neutrino interactions. The distribution of $Y_{e}$ values for particles from simulation B12 is considerably wider than for simulations B13 and B12-sym.

In addition to the high-density lepton captures, neutrino captures at lower densities can also impact $Y_{e}$ at the beginning of nucleosynthesis. We parameterize the neutrino luminosities for the network calculation to determine how much of an impact uncertainties in our neutrino transport approximation have on the nuclear network calculation. In all simulations, higher neutrino luminosities push the particle $Y_{e}$ values more quickly toward the higher end. This is particularly pronounced for neutrino luminosities $L_{\nu}=10^{52} \mathrm{erg} \mathrm{s}^{-1}$ and $L_{\nu}=10^{53} \mathrm{erg} \mathrm{s}^{-1}$. The neutrino luminosities recorded from the tracer particles peak at a few $L_{\nu}=10^{52} \mathrm{erg} \mathrm{s}^{-1}$ (see Figure 4) and are bracketed by the $L_{\nu}=10^{52} \mathrm{erg} \mathrm{s}^{-1}$ and $L_{\nu}=10^{53} \mathrm{erg} \mathrm{s}^{-1}$ constant luminosity cases.

\subsection{Ejecta Composition}

The ejecta properties vary significantly between the simulations. For the $r$-process nucleosynthetic signature of the explosion, the most important factor is how neutron-rich the ejected material is. In Figure 7, we show the distribution of the electron fraction $Y_{e}$ for all particles in the ejected material when the temperature for the particles is last above $5 \mathrm{GK}$. This is representative of $Y_{e}$ at the beginning of neutron-capture nucleosynthesis and leads to different ejecta properties between jet explosions (simulations B13 and B12-sym) and the 3D duallobe explosion (B12). We show results for both the leakage neutrino luminosities and our assumed constant neutrino luminosities. In the case of the leakage neutrino luminosities, the luminosities are also assumed to be constant in the network calculation after the end of the tracer particle data.

For zero neutrino luminosities, the distributions for all simulations are peaked at $Y_{e} \lesssim 0.2$. B12-sym is peaked at lower $Y_{e} \simeq 0.15$ than $\mathrm{B} 12$ at $Y_{e} \simeq 0.21$. The distribution for $\mathrm{B} 12$ is significantly broader than for B12-sym and B13. There are more particles at low $Y_{e}$ values for simulation B12-sym than for B12. This is caused by particles reaching higher densities before they get turned around and swept up in the outflow (see Figure 6). In the $10^{52} \mathrm{erg} \mathrm{s}^{-1}$ luminosity case, neutrino interactions shift the distributions to higher $Y_{e}$ for all simulations. For model B13, where the dwell time of particles in the neutrino field is a factor of $\simeq 10$ shorter than in models B12-sym and B12, this shift is not large, but for simulations B12-sym and B12, the distributions are shifted by almost $\simeq 0.1$. 

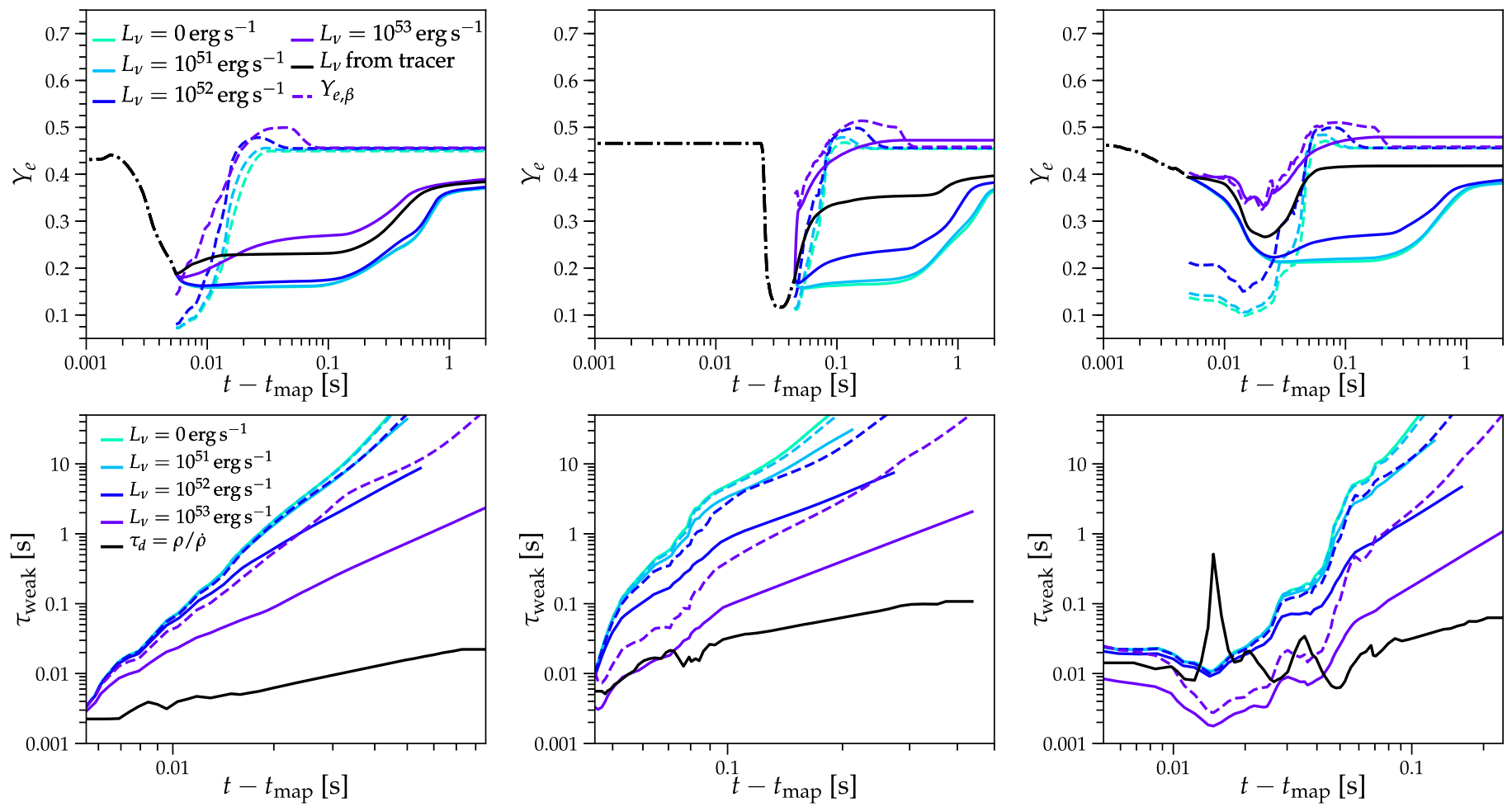

Figure 5. Top row: electron fraction $Y_{e}$ as a function of time after mapping the particles onto simulation B13 (left), B12-sym (center), and B12 (right) for representative particles. Different colored lines indicate results for different neutrino luminosities (assuming $L_{\nu}=L_{\nu_{e}}=L_{\nu_{\bar{e}}}$ ) used in the nuclear reaction network calculation. Black lines indicate results using the neutrino luminosities from the tracer particles advected with the simulations. The dashed lines indicate the evolution of $Y_{e, \beta}$ for each of the fixed neutrino luminosity simulations. The particle in simulation B13 reaches the lowest $Y_{e}$ values, while the particles in simulations B12-sym and B12 turn around at increasing minimum $Y_{e}$ values. The dotted-dashed lines show the evolution of $Y_{e}$ in the tracer particles before the nuclear reaction network calculations begin. Bottom row: weak interaction and dynamical timescales for the same three models. The dashed lines indicate the lepton capture timescale, $\left(\lambda_{e^{-}}+\lambda_{e^{+}}\right)^{-1}$.

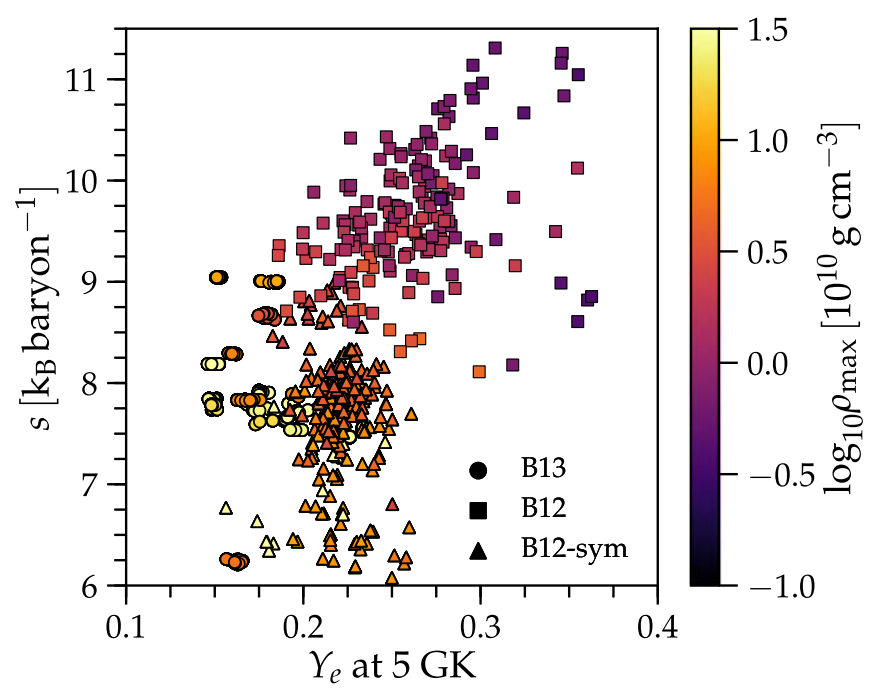

Figure 6. Scatter plot of the electron fraction $Y_{e}$ at $T=5 \mathrm{GK}$ (x-axis) and specific entropy $s$ ( $y$-axis) for select particles from simulations B13 (circles), B12-sym (triangles), and B12 (squares). The symbols are color coded with the maximum density, $\rho_{\max }$, reached.

As a result, there is effectively no material at $Y_{e} \lesssim 0.2$ for simulation B12-sym and no material below $Y_{e} \simeq 0.22$ for simulation B12. The results obtained with the neutrino luminosities from the tracer particles show this effect even more clearly. Here, the effect of neutrino interactions is large enough that even the $Y_{e}$ distribution for simulation B13 is shifted to values of $Y_{e} \gtrsim 0.2$, the distribution for B12-sym is now centered at $Y_{e} \simeq 0.34$, and the $Y_{e}$ distribution for simulation $\mathrm{B} 12$ is shifted to $Y_{e} \simeq 0.36$.

The variations in the distribution of $Y_{e}$ have consequences for the eventual nucleosynthesis, since one must have $Y_{e} \lesssim 0.25$ to make the third $r$-process peak (Lippuner \& Roberts 2015). Figure 8 shows abundance patterns for all three simulations, B13, B12-sym, and B12. We show the fractional abundance pattern averaged over all particles in the ejecta as a function of mass number $A$.

If no neutrino luminosities are taken into account in the nucleosynthesis calculation, we find a robust $r$-process pattern in all three simulations. This is also true for a constant neutrino luminosity of $L_{\nu_{e}}=L_{\overline{\nu_{e}}}=10^{51} \mathrm{erg} \mathrm{s}^{-1}$. For neutrino luminosity $L_{\nu_{e}}=L_{\overline{\nu_{e}}}=10^{52} \mathrm{erg} \mathrm{s}^{-1}$, all simulations still show a robust second $r$-process peak. B13 still has robust third-peak abundances, while B12-sym and B12 have reduced abundances in their third peaks (with B12 seeing the larger reduction). For a neutrino luminosity of $L_{\nu_{e}}=L_{\overline{\nu_{e}}}=10^{53} \mathrm{erg} \mathrm{s}^{-1}$, none of the simulations show significant amounts of material synthesized beyond $A=135$. In all simulations, the reduction in the fractional abundance beyond $A=135$ is accompanied by an overproduction of nuclei with $A<135$ compared to the lower neutrino luminosity cases.

The abundance patterns calculated with the neutrino luminosities, as recorded from the tracer particles, fall in between the $L_{\nu_{e}}=L_{\overline{\nu_{e}}}=10^{52} \mathrm{erg} \mathrm{s}^{-1}$ and $L_{\nu_{e}}=L_{\overline{\nu_{e}}}=10^{53} \mathrm{erg} \mathrm{s}^{-1}$ constant luminosity cases. For simulation B13, material beyond $A>135$ is reduced by a factor of 10 relative to the $L_{\nu}=0$ case, but for 

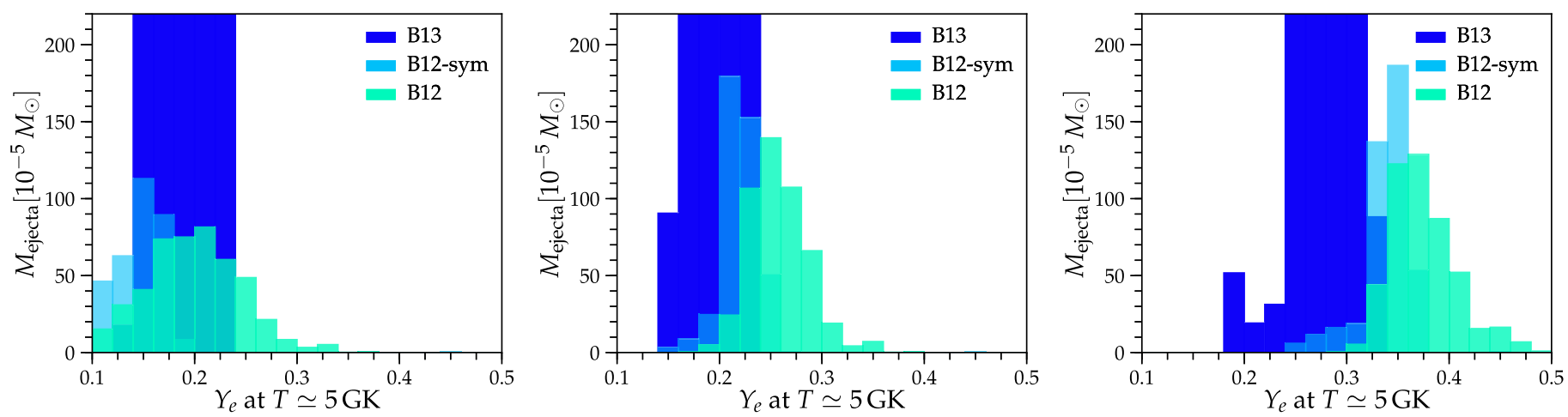

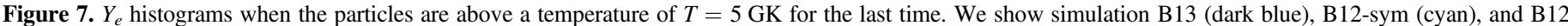

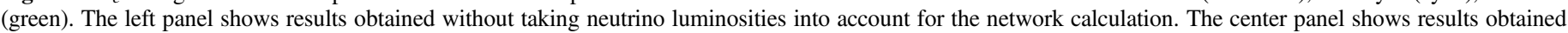

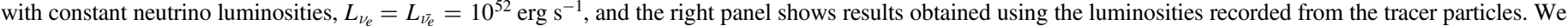
bin $Y_{e}$ in intervals of 0.02 and weigh the $Y_{e}$ statistics with the mass of the ejected particles.

simulations B12-sym and B12, the results with luminosities as recorded from the tracer particles follow the $L_{\nu_{e}}=L_{\overline{\nu_{e}}}=$ $10^{53} \mathrm{erg} \mathrm{s}^{-1}$ case closely. There is no or very little second- or third-peak $r$-process material synthesized.

For a more direct comparison, we show nucleosynthesis calculations with constant neutrino luminosities of $L_{\nu_{e}}=L_{\overline{\nu_{e}}}=$ $10^{52} \mathrm{erg} \mathrm{s}^{-1}$ for simulations B13, B12-sym, and B12 in Figure 9. While model B13 matches the solar abundance pattern well, model B12-sym falls short in the amount of material synthesized beyond $A \simeq 170$ by a factor of a few. For third-peak $r$-process material, the reduction in abundance between models B13 and B12-sym is slightly more than a factor of 10 . For model B12, the reduction in material beyond the second peak is even more severe. Material beyond $A=135$ is underproduced by two orders of magnitude with respect to simulation B13 and the solar abundance pattern. This underproduction is accompanied by an overproduction of nuclei with mass numbers of $50 \leqslant A \leqslant 80$.

We can also compare the elemental abundance patterns produced by our models to the elemental abundance patterns observed in low-metallicity halo stars. In Figure 10, we show the elemental abundances of B12, B12-sym, and B13 along with the observed abundances of the low-metallicity halo stars CS22892-052 (Sneden et al. 2000) and HD122563 (Honda et al. 2006), similar to Nishimura et al. (2017a). CS22892-052 has an abundance pattern that is consistent with the solar $r$-process abundances and, therefore, provides a good match to the abundances of B12-sym and B13. Although the nucleosynthetic pattern of B12 does not match the solar $r$-process abundance pattern or the pattern of CS22892-052, it is reasonably consistent with the incomplete $r$-process abundance pattern of HD122563.

Table 2 summarizes the mass of the total and $r$-process ejecta material for models B13, B12-sym, and B12. The ejecta mass for simulation B13 is an order of magnitude larger than for simulations B12-sym and B12. This is due to the immediate jet launch after core bounce and the propagation speed of $v \simeq 0.15 c$. All of the ejected mass measurements are only lower bounds on the total ejecta mass, since it is still increasing at the end of each of the simulations. Our lowest neutrino luminosity scenario that is still within the uncertainty of the Leakage luminosities from the tracer particles is the constant neutrino luminosity of $10^{52} \mathrm{erg} \mathrm{s}^{-1}$. This acknowledges a factor of up to a few uncertainty in the neutrino luminosities and average energies from the Leakage scheme (E. O'Connor
2018, private communication). For this luminosity, the $r$-process ejecta mass in model B13 is comparable to that found in Winteler et al. (2012) and Nishimura et al. (2015). For neutrino luminosities taken from the tracer particles and for constant neutrino luminosities of $10^{53} \mathrm{erg} \mathrm{s}^{-1}$, the $r$-process ejecta mass is reduced by an order of magnitude. In simulations B12-sym and B12, the $r$-process ejecta mass for our most optimistic scenario is already an order of magnitude smaller than for the same neutrino luminosity in simulation B13. For neutrino luminosities taken from the tracer particles and for constant neutrino luminosities of $10^{53} \mathrm{erg} \mathrm{s}^{-1}$, the $r$-process ejecta mass is effectively zero.

\section{Discussion}

We have studied $r$-process nucleosynthesis from a set of 3D CCSNe simulations. Our models include a full 3D simulation with a precollapse magnetic field of $10^{13} \mathrm{G}(\mathrm{B} 13)$ that is similar in dynamics to the simulation presented in Winteler et al. (2012), a 3D simulation set up to be identical in dynamics to an axisymmetric simulation with a precollapse magnetic field of $10^{12} \mathrm{G}$ (B12-sym) that is similar to the prompt axisymmetric jet explosions in Nishimura et al. (2015), and a full 3D simulation with a precollapse magnetic field of $10^{12} \mathrm{G}$ (B12) as in Mösta et al. (2014b). In our nuclear reaction network calculations, we have included weak interactions to account for interaction of material with neutrinos emitted from the PNS. We have specifically used both parameterized constant neutrino luminosities and the recorded neutrino luminosities from the tracer particles in the simulations.

Our results show that the nucleosynthetic signature of $3 \mathrm{D}$ magnetorotational CCSNe depends on the detailed dynamics of the jet and the neutrino emission from the PNS. Our 3D simulations that include a factor of 10 lower initial magnetic field differ fundamentally from what was anticipated based on either axisymmetric simulations (Nishimura et al. 2015) or 3D simulations of very highly $\left(B \geqslant 5 \times 10^{12} \mathrm{G}\right)$ magnetized progenitor cores (Winteler et al. 2012).

We find that weak interactions in the nuclear reaction network calculations change the nucleosynthetic signatures of all simulations. Including no neutrino luminosities in the network calculation based on simulation B13 produces robust $r$-process abundances consistent with the observed solar abundance pattern and with what Winteler et al. (2012) found. Starting with neutrino luminosities of $5 \times 10^{52} \mathrm{erg} \mathrm{s}^{-1}$, $r$-process material beyond the second peak is reduced in 

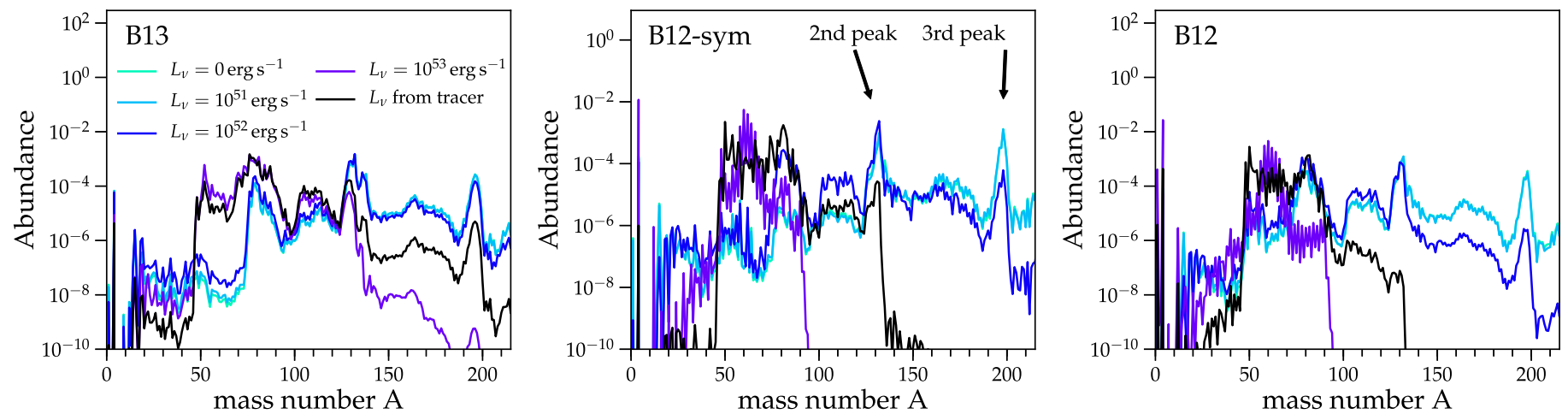

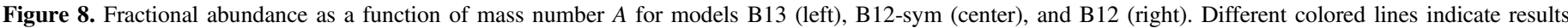

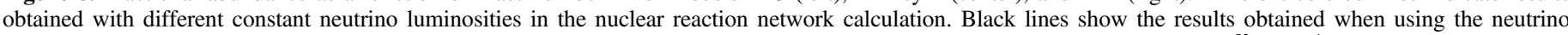

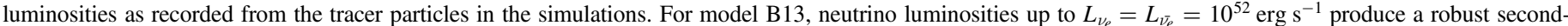

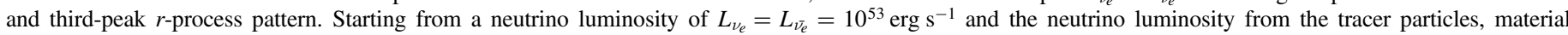

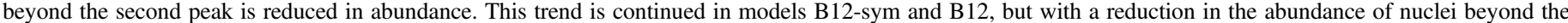

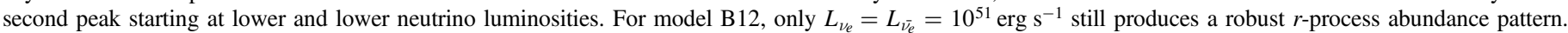

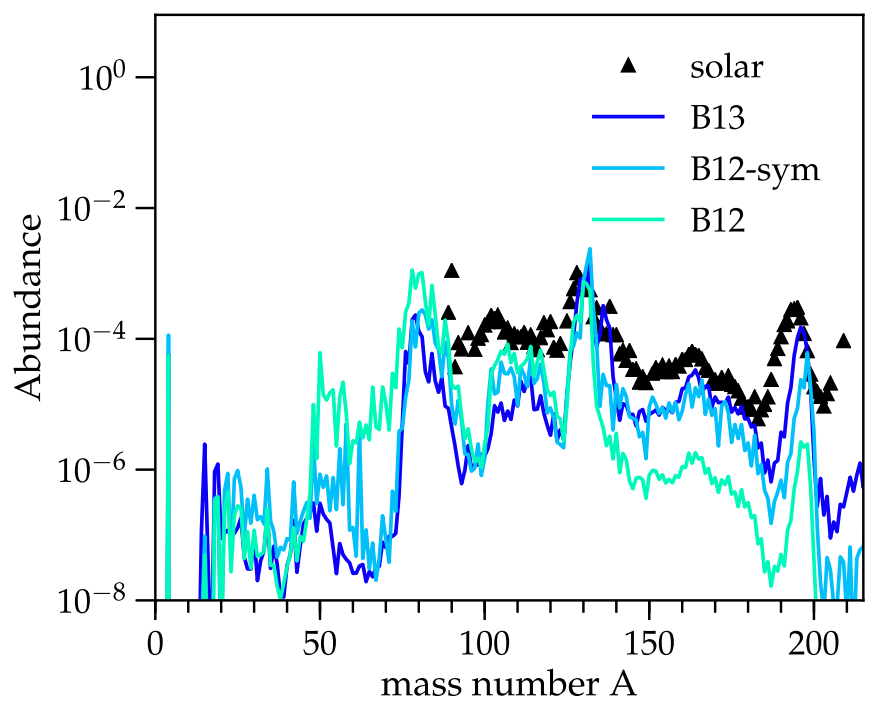

Figure 9. Fractional abundance pattern as a function of mass number $A$ for models B13, B12-sym, and B12. Blue, light blue, and light green show models B13, B12-sym, and B12, respectively, for a constant neutrino luminosity of $L_{\nu_{e}}=L_{\overline{\nu_{e}}}=10^{52} \mathrm{erg} \mathrm{s}^{-1}$ for both electron and electron antineutrinos in the nuclear reaction network calculation. Black markers indicate the solar abundance pattern scaled to match the second $r$-process peak $(A=135)$ for simulation B13. Model B13 reproduces the solar abundance pattern reasonably well, while model B12-sym underproduces third $r$-process peak $(A=195)$ material by more than an order of magnitude. In model B12, all nuclei beyond the second $r$-process peak are reduced in abundance by a factor of $\sim 100$.

abundance by a factor of a few and by an order of magnitude for larger neutrino luminosities. For simulation B12-sym, the reduction in synthesized nuclei beyond the second $r$-process peak starts at neutrino luminosities of $10^{52} \mathrm{erg} \mathrm{s}^{-1}$ but matters mostly for third-peak $r$-process nuclei. For simulation B12, the reduction in abundance of nuclei beyond the second peak is consistently at least a factor of 10 compared to the lower neutrino luminosity calculations. The neutrino luminosities recorded by the tracer particles are typically a few $10^{52} \mathrm{erg} \mathrm{s}^{-1}$ after the initial neutronization burst has subsided after $\sim 20 \mathrm{~ms}$ and hence fall in between the constant luminosity cases of $10^{52}$ and $10^{53} \mathrm{erg} \mathrm{s}^{-1}$. Acknowledging a factor of $\sim 2$ uncertainty in the neutrino luminosities in our simulations, we compare a neutrino luminosity of constant $10^{52} \mathrm{erg} \mathrm{s}^{-1}$ between the three simulations B13, B12-sym, and B12 and the solar abundance pattern as our most optimistic neutrino luminosity scenario. We

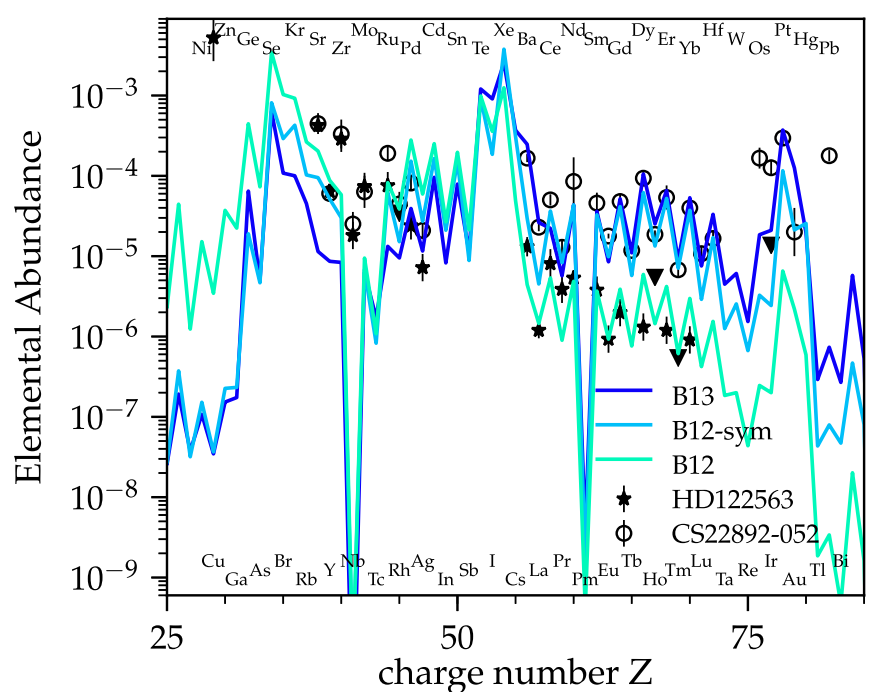

Figure 10. Elemental abundances as a function of nuclear charge $Z$ for models B13, B12-sym, and B12. Blue, light blue, and light green show models B13, B12-sym, and B12, respectively, for a constant neutrino luminosity of $L_{\nu_{e}}=L_{\overline{\nu_{e}}}=10^{52} \mathrm{erg} \mathrm{s}^{-1}$ for both electron and electron antineutrinos in the nuclear reaction network calculation. The black stars represent the observed elemental abundances of the low-metallicity halo star HD122563, while the downward triangles represent observational upper limits (Honda et al. 2006). The open circles represent the scaled elemental abundances of the lowmetallicity halo star CS22892-052 (Sneden et al. 2000). The overall normalization of the abundances patterns of HD122563 and CS22892052 are scaled to minimize the logarithmic residuals with the abundances of B12 and B13, respectively, for elements with charge number greater than 49 .

Table 2

Total and $r$-process Ejecta Masses (Material with $120 \leqslant A \leqslant 249$ ) for the Three Simulations, B13, B12-sym, and B12, for the Four Constant Neutrino Luminosities and the Neutrino Luminosities as Obtained from the Tracer Particles

\begin{tabular}{lccc}
\hline \hline Simulation & B13 & B12-sym & B12 \\
\hline$M_{\mathrm{ej}, \mathrm{tot}}\left(M_{\odot}\right)$ & 0.0356 & 0.0043 & 0.0048 \\
$M_{\mathrm{ej}, \mathrm{r}} L_{\nu}=0 \mathrm{erg} \mathrm{s}^{-1}\left(M_{\odot}\right)$ & 0.0337 & 0.0042 & 0.0038 \\
$M_{\mathrm{ej}, \mathrm{r}} L_{\nu}=10^{51} \mathrm{erg} \mathrm{s}^{-1}\left(M_{\odot}\right)$ & 0.0336 & 0.0042 & 0.0037 \\
$M_{\mathrm{ej}, \mathrm{r}} L_{\nu}=10^{52} \mathrm{erg} \mathrm{s}^{-1}\left(M_{\odot}\right)$ & 0.0320 & 0.0034 & 0.0018 \\
$M_{\mathrm{ej}, \mathrm{r}} L_{\nu}$ from tracer $\left(M_{\odot}\right)$ & 0.0038 & $5.4 \times 10^{-5}$ & $4.0 \times 10^{-7}$ \\
$M_{\mathrm{ej}, \mathrm{r}} L_{\nu}=10^{53} \mathrm{erg} \mathrm{s}^{-1}\left(M_{\odot}\right)$ & 0.0012 & 0.0 & 0.0
\end{tabular}

Note. Masses are in solar masses, $M_{\odot}$. 
find a robust second and third-peak abundance pattern only for simulation B13. Simulation B12-sym shows an underproduction of nuclei beyond $A=170$ by a factor of a few. For the full $3 \mathrm{D}$ simulation $\mathrm{B} 12$, we find that nuclei beyond the second $r$-process peak are underproduced by a factor of 100 compared to solar abundances.

Our results show that the realistic 3D dynamics of magnetorotationally driven CCSNe change their $r$-process nucleosynthetic signatures. The different explosion dynamics lead to ejecta material probing different regions of the engine driving the explosion. In simulation B13, material from the smallest radii gets entrained in the outflow, while material in the outflows of simulations B12-sym and B12 originates at larger radii and lower densities. This leads to less neutron-rich material being entrained in the outflows for simulation B12. In addition, the dwell time of ejecta material in the vicinity of the PNS for simulations B12-sym and B12 is a factor of $\simeq 10$ longer than for simulation B13. This causes the $Y_{e}$ distribution of the ejecta at the onset of $r$-process nucleosynthesis to shift to higher $Y_{e}$ for simulations B12-sym and B12. This is especially true for simulation B12, for which the distribution of $Y_{e}$ in the ejecta at the onset of $r$-process nucleosynthesis is peaked at $\sim 0.28$ and is broader than for simulations B13 and B12-sym. For the full 3D dynamics of the explosion in simulation B12, the mass of ejected $r$-process material is an order of magnitude smaller even for our most optimistic scenario of a constant neutrino luminosity of $10^{52} \mathrm{erg} \mathrm{s}^{-1}$. For neutrino luminosities obtained from the tracers particles, the ejected $r$-process mass is only $\sim 10^{-7} M_{\odot}$. Most importantly, third-peak material is a factor of 100 less abundant when compared with perhaps unrealistic jet explosions, like simulations B13 and B12-sym.

Our results suggest that the only viable channel for a robust $r$-process pattern is via an immediate jet explosion at core bounce, although an incomplete $r$-process abundance pattern consistent with the abundance pattern of low-metallicity halo star HD122563 is produced in a delayed jet-driven explosion. Such an explosion is extremely effective at funneling material into the jet-driven outflow, leaving little time for weak interactions to push the ejected material to higher electron fraction values. For this case, and as in Winteler et al. (2012), we find a robust $r$-process abundance pattern consistent with observed solar abundances. The immediate jet launch at core bounce requires $10^{16} \mathrm{G}$ of a large-scale toroidal field and a poloidal field of similar strength to stabilize the outflow against the kink instability. This field can be generated via amplification by the MRI and a dynamo process, but the amplification will take at least 10 spin periods of the PNS (Mösta et al. 2015). For realistic precollapse iron cores with magnetic fields not in excess of $\sim 10^{8}-10^{9} \mathrm{G}$, this amplification timescale is even longer. In addition, the MRI and dynamo action will likely saturate at field strengths of no more than $\sim 10^{15} \mathrm{G}$ (Rembiasz et al. 2016) for both the poloidal and toroidal components, falling short of the required ultra-strong poloidal field required to stabilize the jet. Therefore, the magnetic field in simulation B13 (and similarly the fields in the simulation presented in Winteler et al. 2012) cannot be assumed to be delivered by this amplification channel. The only viable channel to achieve field strengths as in simulation B13 is thus the collapse of iron cores with sufficiently strong $\left(B \gtrsim 10^{13} \mathrm{G}\right)$ precollapse poloidal fields, which are likely unrealistic. Nishimura et al. (2017b) have studied $r$-process nucleosynthesis from axisymmetric simulations of MRI-driven explosions and have also found only weak $r$-process patterns.

The reduced abundance of ejecta material beyond the second peak from our simulations changes the predicted yield of $r$-process material per event for magnetorotational supernovae. The masses of $r$-process elements ejected from simulations B13 and B12-sym are consistent with the assumptions of previous studies of the role of magnetorotational supernovae in galactic chemical evolution (Nishimura et al. 2015; Wehmeyer et al. 2015); however, the $r$-process ejecta mass from simulation B12 is significantly lower. This will have to be taken into account when studying galactic chemical evolution and the role of magnetorotational supernovae in early $r$-process enrichment. It is particularly important given recent evidence that neutron star mergers may not be able to explain $r$-process enrichment in the lowest metallicity stars (e.g., Casey \& Schlaufman 2017) and evidence for multiple, distinct enrichment channels in dwarf galaxies (Tsujimoto et al. 2017).

The authors would like to thank D. Kasen, E. Quataert, and D. Radice for discussions. This research was partially supported by NSF grants AST-1212170, CAREER PHY-1151197, OAC-1550514, and OCI-0905046. P.M. acknowledges support by NASA through Einstein Fellowship grant PF5-160140. This work was enabled in part by the NSF under Grant No. PHY1430152 (JINA Center for the Evolution of the Elements). The simulations were carried out on XSEDE resources under allocation TG-AST160049 and on NSF/NCSA BlueWaters under NSF award PRAC OCI-0941653. This paper has been assigned Yukawa Institute for Theoretical Physics report number YITP-17-129 and LANL Report number LA-UR-1731278.

Software: Einstein Toolkit (Löffler et al. 2012; Mösta et al. 2014a), SkyNet (Lippuner \& Roberts 2017), REACLIB (Cyburt et al. 2010), Matplotlib (Hunter 2007).

\section{ORCID iDs}

Philipp Mösta (iD https://orcid.org/0000-0002-9371-1447 Christian D. Ott (iD https://orcid.org/0000-0003-4993-2055 Jonas Lippuner (iD https://orcid.org/0000-0002-5936-3485 Erik Schnetter (i) https://orcid.org/0000-0002-4518-9017

\section{References}

Akiyama, S., Wheeler, J. C., Meier, D. L., \& Lichtenstadt, I. 2003, ApJ, 584,954

Arcones, A., Martínez-Pinedo, G., Roberts, L. F., \& Woosley, S. E. 2010, A\&A, 522, A25

Balbus, S. A., \& Hawley, J. F. 1991, ApJ, 376, 214

Bisnovatyi-Kogan, G. S. 1970, Astron. Zh., 47, 813

Burrows, A., Dessart, L., Livne, E., Ott, C. D., \& Murphy, J. 2007, ApJ, 664,416

Casey, A. R., \& Schlaufman, K. C. 2017, ApJ, 850, 179

Cyburt, R. H., Amthor, A. M., Ferguson, R., et al. 2010, ApJS, 189, 240

Einfeldt, B. 1988, in Proc. Sixteenth Int. Symp., Shock Tubes and Waves (VCH: New York), 671

Fuller, G. M., Fowler, W. A., \& Newman, M. J. 1982, ApJS, 48, 279

Halevi, G., \& Mösta, P. 2018, MNRAS, 477, 2366

Heger, A., Langer, N., \& Woosley, S. E. 2000, ApJ, 528, 368

Hoffman, R. D., Woosley, S. E., \& Qian, Y.-Z. 1997, ApJ, 482, 951

Honda, S., Aoki, W., Ishimaru, Y., Wanajo, S., \& Ryan, S. G. 2006, ApJ, 643,1180

Hotokezaka, K., Kiuchi, K., Kyutoku, K., et al. 2013, PhRvD, 87, 024001

Hunter, J. D. 2007, CSE, 9, 90

Kruskal, M., \& Tuck, J. L. 1958, Proc.R. Soc. Lond., 245, 222

Langanke, K., \& Martínez-Pinedo, G. 2000, NuPhA, 673, 481 
Lattimer, J. M., \& Swesty, F. D. 1991, NuPhA, 535, 331

LeBlanc, J. M., \& Wilson, J. R. 1970, ApJ, 161, 541

Lippuner, J., \& Roberts, L. F. 2015, ApJ, 815, 82

Lippuner, J., \& Roberts, L. F. 2017, ApJS, 233, 18

Löffler, F., Faber, J., Bentivegna, E., et al. 2012, Class. Quantum Grav., 29 115001

Meier, D. L., Epstein, R. I., Arnett, W. D., \& Schramm, D. N. 1976, ApJ, 204, 869

Meyer, B. S., \& Brown, J. S. 1997, ApJS, 112, 199

Mösta, P., Mundim, B. C., Faber, J. A., et al. 2014a, Class. Quantum Grav., 31 015005

Mösta, P., Ott, C. D., Radice, D., et al. 2015, Natur, 528, 376

Mösta, P., Richers, S., Ott, C. D., et al. 2014b, ApJL, 785, L29

Nishimura, N., Hirschi, R., Rauscher, T., Murphy, A. S. J., \& Cescutti, G. 2017a, MNRAS, 469, 1752

Nishimura, N., Sawai, H., Takiwaki, T., Yamada, S., \& Thielemann, F.-K. 2017b, ApJL, 836, L21

Nishimura, N., Takiwaki, T., \& Thielemann, F.-K. 2015, ApJ, 810, 109

Obergaulinger, M., \& Aloy, M. Á 2017, MNRAS, 469, L43
Obergaulinger, M., Cerdá-Durán, P., Müller, E., \& Aloy, M. A. 2009, A\&A, 498, 241

O’Connor, E., \& Ott, C. D. 2010, Class. Quantum Grav, 27, 114103

Oda, T., Hino, M., Muto, K., Takahara, M., \& Sato, K. 1994, ADNDT, 56, 231

Ott, C. D., Abdikamalov, E., O’Connor, E., et al. 2012, PhRvD, 86, 024026

Ott, C. D., Burrows, A., Thompson, T. A., Livne, E., \& Walder, R. 2006, ApJS, 164, 130

Qian, Y.-Z., \& Woosley, S. E. 1996, ApJ, 471, 331

Reisswig, C., Haas, R., Ott, C. D., et al. 2013, Phys. Rev.D., 87, 064023

Rembiasz, T., Guilet, J., Obergaulinger, M., et al. 2016, MNRAS, 460, 3316

Sneden, C., Cowan, J. J., Ivans, I. I., et al. 2000, ApJL, 533, L139

Takiwaki, T., \& Kotake, K. 2011, ApJ, 743, 30

Takiwaki, T., Kotake, K., \& Suwa, Y. 2012, ApJ, 749, 98

Tchekhovskoy, A., McKinney, J. C., \& Narayan, R. 2007, MNRAS, 379, 469 Tóth, G. 2000, JCoPh, 161, 605

Tsujimoto, T., Yokoyama, T., \& Bekki, K. 2017, ApJL, 835, L3

Wehmeyer, B., Pignatari, M., \& Thielemann, F.-K. 2015, MNRAS, 452, 1970

Wheeler, J. C., Meier, D. L., \& Wilson, J. R. 2002, ApJ, 568, 807

Winteler, C., Käppeli, R., Perego, A., et al. 2012, ApJL, 750, L22 